\title{
Usefulness of Transesophageal Echocardiography for Predicting Covert Paroxysmal Atrial Fibrillation in Patients with Embolic Stroke of Undetermined Source
}

\author{
Yuichiro Ohya ${ }^{a}$ Masato Osaki ${ }^{a}$ Shigeru Fujimoto ${ }^{b}$ Juro Jinnouchi ${ }^{a}$

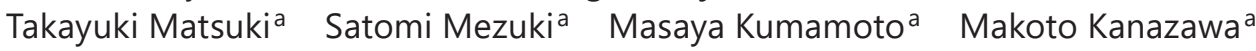 \\ Naoki Tagawa ${ }^{a}$ Tetsuro Agoc ${ }^{c}$ Takanari Kitazonoc Shuji Arakawa ${ }^{c}$ \\ a Department of Cerebrovascular Medicine, Stroke Center, Steel Memorial Yawata Hospital, \\ Kitakyushu, Japan; ${ }^{b}$ Division of Neurology, Department of Medicine, Jichi Medical \\ University, Tochigi, Japan; ' Department of Medicine and Clinical Science, Graduate School \\ of Medical Sciences, Kyushu University, Fukuoka, Japan
}

Keywords

Atrial fibrillation - Embolic stroke of undetermined source - Transesophageal echocardiography

\section{Abstract}

Background: Covert paroxysmal atrial fibrillation (CPAF) is a major cause of embolic stroke of undetermined source (ESUS). However, detecting PAF during hospitalization in these patients is difficult. Objectives: This study aimed to determine whether findings of transesophageal echocardiography (TEE) during hospitalization are associated with later detection of PAF in patients with ESUS. Method: We retrospectively studied 348 patients with ESUS who were admitted to our hospital within 1 week of onset. These patients met the criteria of ESUS, underwent TEE during hospitalization, and were followed up for at least 1 year. Results: We found PAF in 35 (10.0\%) patients. In patients with PAF, spontaneous echo contrast (SEC) and low left atrial appendage flow (LAAF) by TEE and enlargement of the left atrial dimension (LAD) by transthoracic echocardiography were identified more frequently compared with those who did not have PAF. In multivariate analysis, SEC and an LAD $\geq 42 \mathrm{~mm}$ were independently associated with later detection of PAF $(p<0.05)$. An association of LAAF $<46.9 \mathrm{~cm} / \mathrm{s}$ and PAF was marginal $(p=0.09)$. The specificity of the combined finding of SEC and/or LAAF with that of LAD increased up to $90 \%$, while that of LAD alone was $70 \%$. Conclusions: The findings of TEE during hospitalization may be useful for identifying patients at increased risk of CPAF in patients with ESUS. 


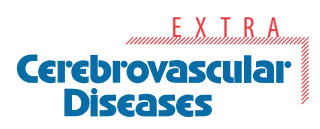

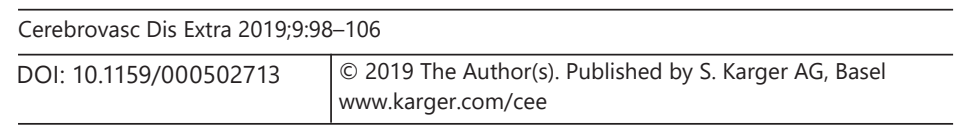

Ohya et al.: TEE Findings for Predicting CPAF in ESUS

\section{Introduction}

Approximately $25 \%$ of all ischemic strokes are cryptogenic [1]. Among them, the clinical entity of embolic stroke of undetermined source (ESUS) has been recently established and is expected to be useful for determining how antithrombotic therapy is performed [1]. However, ESUS is simply defined as nonlacunar brain infarction without proximal arterial stenoocclusive lesion or cardioembolic sources. Therefore, ESUS can include heterogenous pathogenesis, such as covert paroxysmal atrial fibrillation (CPAF), minor-risk potential cardioembolic sources, arteriogenic/aortogenic embolism, paradoxical embolism, cancer-associated embolism, and undetermined embolism (unidentified or two potential embolic sources). Therefore, antithrombotic therapy (i.e., anticoagulation or antiplatelet) should be considered on the basis of the individual pathogenesis. An example of this situation is that ESUS with CPAF should be treated with anticoagulants for secondary prevention. However, detecting paroxysmal atrial fibrillation (PAF) during hospitalization is often difficult, even with continuous electrocardiographic (ECG) monitoring. Prolonged rhythm monitoring, including an insertable cardiac monitor, is currently available and may contribute to the detection of PAF $[2,3]$.

Recent studies have suggested that transesophageal echocardiography (TEE) may be useful for determining the pathogenesis in patients with ESUS $[4,5]$. TEE can be used to evaluate plaque formation of the aortic arch, which can cause aortogenic embolism, and the presence of a patent foramen ovale ( $\mathrm{PFO}$ ), which can potentially cause paradoxical embolism. However, PFO is observed in up to $30 \%$ of healthy controls [4, 5]. Furthermore, TEE enables evaluation of left atrial function in detail, which cannot be evaluated by transthoracic echocardiography (TTE). Spontaneous echo contrast (SEC), which is an echogenic swirling pattern of blood flow in the left atrium [6], and left atrial appendage flow (LAAF), which is known to be associated with appendage thrombus [7], may be highly associated with left atrial function. Decreased left atrial function may be associated with the presence of atrial fibrillation (AF). Therefore, we hypothesize that abnormal findings of the left atrium as detected by TEE predict the presence of CPAF in patients with ESUS.

In the present study, we retrospectively examined whether TEE findings in patients with the acute phase of ESUS during hospitalization are associated with later detection of PAF in a follow-up of longer than 1 year.

\section{Materials and Methods}

This study was approved by our institutional ethics review board. We obtained general written informed consent about the use of clinical data for educations and researches from all patients who were admitted to our hospital for acute stroke, although it was not specific for this study.

\section{Patients}

We retrospectively evaluated 1,244 patients who developed ischemic stroke or transient ischemic attack. These patients were admitted to our hospital within 1 week of onset from March 2008 to April 2014. We suspected 732 patients of having embolic stroke because of their symptoms and the site of ischemic stroke, such as sudden onset, and cortical or subcortical lesions. Of these patients, 491 underwent TEE, and 354 patients met the criteria of ESUS proposed by Hart et al. [1]. Finally, 348 patients were followed up for longer than 1 year. 


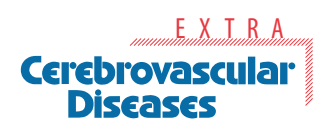

\begin{tabular}{l|l}
\hline Cerebrovasc Dis Extra 2019;9:98-106 \\
\hline DOI: 10.1159/000502713 & $\begin{array}{l}\text { ○ 2019 The Author(s). Published by S. Karger AG, Basel } \\
\text { www.karger.com/cee }\end{array}$ \\
\hline
\end{tabular}

Ohya et al.: TEE Findings for Predicting CPAF in ESUS

\section{Clinical Assessment}

The following cerebrovascular risk factors were investigated: hypertension (systolic blood pressure $\geq 140 \mathrm{~mm} \mathrm{Hg}$ or diastolic blood pressure $\geq 90 \mathrm{~mm} \mathrm{Hg}$, or current treatment with antihypertensive medication), diabetes mellitus (fasting plasma glucose levels $\geq 126$ $\mathrm{mg} / \mathrm{dL}$ or plasma glucose levels $\geq 200 \mathrm{mg} / \mathrm{dL}$ at any time, and hemoglobin $A_{1 \mathrm{c}} \geq 6.5 \%$ or current treatment with antidiabetic medication), hypercholesterolemia (serum low-density lipoprotein-cholesterol levels $\geq 3.62 \mathrm{mmol} / \mathrm{L}$ or current treatment with antihyperlipidemic medication), end-stage renal failure (creatinine clearance $\leq 15 \mathrm{~mL} / \mathrm{min}$ ), smoking (previous or current cigarette smoking), drinking (current habitual drinking), and a history of stroke and heart disease (ischemic or valvular heart disease).

\section{Echocardiography}

TTE and TEE were performed. Data of TTE were obtained using a commercially available ultrasound system (model iU22; Philips, Eindhoven, the Netherlands) equipped with a $2.5-\mathrm{MHz}$ phased array transducer for TTE. Left atrial dimension (LAD) was measured using the M mode or 2-dimensional echocardiography. LAD was measured from the posterior aortic wall to the posterior left atrial wall in the parasternal long axis view at end-ventricular systole [8]. Left ventricular mass was estimated by the Devereux equation. A commercially available real-time 2-dimensional echocardiography system (model iU22; Philips) equipped with a $5.0-\mathrm{MHz}$ phased array omniplane transesophageal transducer was used for TEE. TEE was performed within at least 7 days after onset of stroke. First, the left atrium was observed, and the presence of SEC, thrombus, and valvular strands was assessed. SEC was defined as an echogenic swirling pattern of blood flow in the left atrium at every $0,30,60$, and 90 degrees, and this was distinct from white noise artifacts [6]. We then measured LAAF and mobility of the atrial septum.

We adopted the lowest value of inflow or outflow velocity as LAAF. Atrial septal aneurysm was defined as $\geq 13 \mathrm{~mm}$ of mobility of the atrial septum $[9,10]$. The presence of a right-to-left shunt, such as PFO or pulmonary arteriovenous fistula, was then examined by a microbubble study according to recommendations for echocardiography by the European Society of Cardiology [11]. Briefly, tiny bubbles were formed by shaking sterile salt solution and injecting it into an antecubital vein. PFO and pulmonary arteriovenous fistula were defined as appearance of bubbles in the left atrium within 3 heart beats or after 4 heart beats, respectively. Finally, the aortic arch was observed in the transverse and sagittal views. Maximum intima-media thickness $>4.0 \mathrm{~mm}$ and the presence of ulcerative or mobile plaques were regarded as complex aortic arch atheroma [6].

\section{Follow-Up Examination to Detect PAF}

The mean length of stay in hospital for ischemic stroke or transient ischemic attack was 14 days (9-19 days). During hospitalization, ECG monitoring was performed in all patients. Holter ECG was also performed in 97\% (337/348 patients) of patients at least once.

Patients were followed up at our outpatient clinic and affiliated primary care physicians' offices approximately once a month after discharge from our hospital. Physicians checked the patients' pulse and asked patients about complaints of palpitations and irregularity of their pulse. Occasional ECG or Holter ECG at a physician's request was performed to detect PAF. We examined whether AF had been detected during follow-up periods from the medical records. "PAF" which we described as the outcome could include CPAF and developing AF, because we could not distinguish these states.

\section{Statistical Analysis}

The two-tailed $t$ test, Wilcoxon two-tailed test, and $\chi^{2}$ test were used to compare parameters between patients with and without PAF. A two-tailed $p<0.05$ was considered to indicate 
Table 1. Patients' characteristics

\begin{tabular}{|c|c|c|c|c|}
\hline & \multirow{2}{*}{$\begin{array}{l}\text { Total } \\
(n=348)\end{array}$} & \multicolumn{2}{|c|}{ Paroxysmal atrial fibrillation } & \multirow[t]{2}{*}{$p$} \\
\hline & & $\begin{array}{l}\text { detected } \\
(n=35)\end{array}$ & $\begin{array}{l}\text { not detected } \\
(n=313)\end{array}$ & \\
\hline Age, years & $72.2 \pm 11.5$ & $75.4 \pm 7.2$ & $71.9 \pm 11.8$ & 0.09 \\
\hline Men & $200(57.5 \%)$ & $17(48.6 \%)$ & $183(58.5 \%)$ & 0.26 \\
\hline Hypertension & $271(77.9 \%)$ & $29(82.9 \%)$ & $242(77.3 \%)$ & 0.45 \\
\hline Diabetes mellitus & $103(29.6 \%)$ & $8(22.9 \%)$ & $95(30.4 \%)$ & 0.36 \\
\hline Hypercholesterolemia & $187(53.7 \%)$ & $16(45.7 \%)$ & $171(54.6 \%)$ & 0.32 \\
\hline End-stage renal failure & $19(5.5 \%)$ & $3(8.6 \%)$ & $16(5.1 \%)$ & 0.39 \\
\hline Smoking & $174(50.0 \%)$ & $13(37.1 \%)$ & $161(51.4 \%)$ & 0.11 \\
\hline Drinking & $140(40.2 \%)$ & $15(42.9 \%)$ & $125(39.9 \%)$ & 0.74 \\
\hline History of heart disease & $101(29.0 \%)$ & $12(34.3 \%)$ & $89(28.4 \%)$ & 0.47 \\
\hline History of stroke & $68(19.5 \%)$ & $5(14.3 \%)$ & $63(20.1 \%)$ & 0.41 \\
\hline NIHSS score on admission & 2 (IQR 0-4) & 2 (IQR 0-4) & 2 (IQR 0-4) & 0.80 \\
\hline
\end{tabular}

NIHSS, National Institute of Health Stroke Scale; IQR, interquartile range. Data for age are shown as means $\pm \mathrm{SD}$

statistical significance. The best cutoff points of LAD and LAAF for predicting PAF were decided by receiver operating characteristic curve analysis retrospectively. We then examined which echocardiographic parameters were associated with the presence of PAF by multivariate analysis. Analyzed parameters included age, sex, hypertension, diabetes mellitus, hypercholesterolemia, end-stage renal failure, smoking, drinking, a history of stroke, a history of heart disease, SEC, LAAF $<46.9 \mathrm{~cm} / \mathrm{s}$, and LAD $\geq 42 \mathrm{~mm}$. We created a model of combined parameters using echocardiographic findings with $p<0.10$ in multivariate analysis and evaluated sensitivity and specificity of the model. The data were analyzed using JMP 12.2.0 software (SAS Institute, Cary, NC, USA).

\section{Results}

\section{Patients' Characteristics}

A total of 348 patients who were diagnosed with ESUS and underwent TEE were included in this study. The patients' characteristics are shown in Table 1 . The mean age of patients was $72.2 \pm 11.5$ years, and $57.5 \%$ were men. The mean follow-up period was 60.0 months (44.080.8 months). AF was detected in $35(10.0 \%)$ patients during the follow-up period. Risk factors, a medical history of heart disease and stroke, and the National Institutes of Health Stroke Scale score on admission were not different between patients with and without PAF.

\section{Echocardiographic Findings}

Abnormal findings were observed in 291 (83.6\%) patients by TEE. Complex aortic arch atheroma was most frequently found, followed by right-to-left shunt, including PFO and pulmonary arteriovenous fistula (Table 2). However, these findings were equally observed between patients with and without PAF. In contrast, SEC was more frequently observed in patients with PAF than in those without PAF $(p<0.0001)$. Furthermore, patients with PAF had significantly lower LAAF than did those without PAF $(p<0.05)$. The best cutoff point of LAAF for predicting PAF was $46.9 \mathrm{~cm} / \mathrm{s}$ by receiver operating characteristic curve analysis. 
Table 2. Echocardiographic findings

\begin{tabular}{|c|c|c|c|c|}
\hline & \multirow{2}{*}{$\begin{array}{l}\text { Total } \\
(n=348)\end{array}$} & \multicolumn{2}{|l|}{ Paroxysmal atrial fibrillation } & \multirow[t]{2}{*}{$p$} \\
\hline & & $\begin{array}{l}\text { detected } \\
(n=35)\end{array}$ & $\begin{array}{l}\text { not detected } \\
(n=313)\end{array}$ & \\
\hline \multicolumn{5}{|l|}{ TEE findings } \\
\hline Complex aortic arch atheroma & $213(61.2 \%)$ & $25(71.4 \%)$ & $188(60.1 \%)$ & 0.19 \\
\hline Mobile plaque & $23(6.6 \%)$ & $4(11.4 \%)$ & $19(6.1 \%)$ & 0.23 \\
\hline Ulcer & $87(25.0 \%)$ & $8(22.9 \%)$ & $79(25.2 \%)$ & 0.76 \\
\hline Patent foramen ovale & $50(14.4 \%)$ & $2(5.7 \%)$ & $48(15.3 \%)$ & 0.12 \\
\hline Pulmonary arteriovenous fistula & $27(7.8 \%)$ & $2(5.7 \%)$ & $25(8.0 \%)$ & 0.63 \\
\hline Spontaneous echo contrast & $36(10.3 \%)$ & $11(31.4 \%)$ & $25(8.0 \%)$ & $<0.0001$ \\
\hline Left atrial thrombus & $4(1.2 \%)$ & $1(2.9 \%)$ & $3(1.0 \%)$ & 0.32 \\
\hline Atrial septal aneurysm & $39(11.2 \%)$ & $1(2.9 \%)$ & $38(12.1 \%)$ & 0.10 \\
\hline Valvular strands & $92(26.4 \%)$ & $6(17.1 \%)$ & $86(27.5 \%)$ & 0.19 \\
\hline Left atrial appendage flow, $\mathrm{cm} / \mathrm{s}$ & 67.0 (IQR 51.6-82.3) & 56.6 (IQR 32.9-80.7) & 67.9 (IQR 53.2-82.3) & 0.02 \\
\hline$<25.0 \mathrm{~cm} / \mathrm{s}$ & $8(2.3 \%)$ & $4(11.4 \%)$ & $4(1.3 \%)$ & 0.0001 \\
\hline$<46.9 \mathrm{~cm} / \mathrm{s}$ & $63(18.1 \%)$ & $14(40.0 \%)$ & $49(15.7 \%)$ & 0.0004 \\
\hline Others & $3(0.9 \%)$ & $0(0 \%)$ & $3(1.0 \%)$ & 0.56 \\
\hline No findings & $57(16.4 \%)$ & $5(14.3 \%)$ & $52(16.6 \%)$ & 0.72 \\
\hline \multicolumn{5}{|l|}{ TTE findings } \\
\hline Left atrial dimension, mm & $38.7 \pm 5.9$ & $41.7 \pm 4.9$ & $38.3 \pm 5.9$ & 0.001 \\
\hline Left ventricular mass index & 145.0 (IQR 118.2-174.7) & 153.6 (IQR 126.6-184.0) & 144.2 (IQR 117.1-174.6) & 0.33 \\
\hline Fractional shortening, \% & 40.9 (IQR 35.4-45.9) & 41.0 (IQR 35.7-45.5) & 40.9 (IQR 35.2-46.0) & 0.89 \\
\hline Ejection fraction & 71.0 (IQR 64.0-77.0) & 70.0 (IQR 65.0-76.0) & 71.5 (IQR 64.0-77.3) & 0.65 \\
\hline
\end{tabular}

IQR, interquartile range; TEE, transesophageal echocardiography; TTE, transthoracic echocardiography. Other TEE findings included ventricular septal defect $(n=1)$, atrial septal defect $(n=1)$, cardiac tumor $(n=1)$, Chiari network $(n=1)$, and right atrial thrombus $(n=1)$. Data for left atrial dimension are shown as means \pm SD.

Table 3. Associations between clinical parameters and detection of paroxysmal atrial fibrillation

\begin{tabular}{llll}
\hline & OR & $95 \%$ CI & $p$ \\
\hline Left atrial dimension $\geq 42 \mathrm{~mm}$ & 3.64 & $1.64-8.34$ & 0.002 \\
Spontaneous echo contrast & 3.60 & $1.29-9.80$ & 0.01 \\
Left atrial appendage flow $<46.9 \mathrm{~cm} / \mathrm{s}$ & 2.27 & $0.89-5.57$ & 0.09 \\
Age & 0.99 & $0.04-1.03$ & 0.72 \\
Men & 1.41 & $0.46-4.42$ & 0.55 \\
Hypertension & 0.62 & $0.19-1.71$ & 0.37 \\
Diabetes mellitus & 1.12 & $0.45-3.02$ & 0.38 \\
Hypercholesterolemia & 1.43 & $0.64-3.23$ & 0.37 \\
End-stage renal failure & 0.48 & $0.11-2.64$ & 0.18 \\
Smoking & 1.99 & $0.73-5.42$ & 0.28 \\
Drinking & 0.59 & $0.23-1.52$ & 0.55 \\
History of heart disease & 1.32 & $0.54-3.41$ & 0.37 \\
History of stroke & 1.64 & $0.57-5.54$ & \\
\hline
\end{tabular}

OR, odds ratio; 95\% CI, 95\% confidence interval. Left atrial dimension was measured by transthoracic echocardiography, and the other two parameters (spontaneous echo contrast and left atrial appendage flow) were measured by transesophageal echocardiography. Analyzed parameters included age, sex, hypertension, diabetes mellitus, hypercholesterolemia, end-stage renal failure, smoking, drinking, a history of stroke, a history of heart disease, spontaneous echo contrast, left atrial appendage flow $<46.9 \mathrm{~cm} / \mathrm{s}$, and left atrial dimension $\geq 42 \mathrm{~mm}$. 
Table 4. Sensitivity and specificity of parameters

\begin{tabular}{lllll}
\hline & Sensitivity & Specificity & PPV & NPV \\
\hline Parameters & & & & \\
LAD $\geq 42 \mathrm{~mm}$ & 0.60 & 0.70 & 0.19 & 0.94 \\
LAAF $<46.9 \mathrm{~cm} / \mathrm{s}$ & 0.40 & 0.84 & 0.22 & 0.93 \\
SEC & 0.31 & 0.92 & 0.31 & 0.92 \\
LAD and LAAF & 0.31 & 0.95 & 0.42 & 0.93 \\
LAD and SEC & 0.17 & 0.96 & 0.35 & 0.91 \\
All three factors & 0.17 & 0.99 & 0.60 & 0.91 \\
\hline
\end{tabular}

LAD, left atrial dimension in transthoracic echocardiography; LAAF, left atrial appendage flow in transesophageal echocardiography; SEC, spontaneous echo contrast in transesophageal echocardiography; PPV, positive predictive value; NPV, negative predictive value. All three factors: LAD, LAAF, and SEC.

Other findings or parameters were not significantly different between patients with and without PAF.

For parameters of TTE, LAD, left ventricular mass index, percent fractional shortening, and ejection fraction were evaluated. Only LAD was significantly higher in patients with PAF than in those without PAF ( $p<0.005)$. The best cutoff point of LAD was $42 \mathrm{~mm}$ by receiver operating characteristic curve analysis.

\section{Predictors of Echocardiographic Findings for PAF}

Multivariate analyses were performed to exclude possible interaction among echocardiographic and clinical findings. SEC and LAD $\geq 42 \mathrm{~mm}$ were independently associated with PAF ( $p<0.05$, Table 3). An association of LAAF $<46.9 \mathrm{~cm} / \mathrm{s}$ and PAF was marginal ( $p=0.09$, Table 3).

The sensitivity and specificity of three echocardiographic findings (SEC, LAAF $<46.9$ $\mathrm{cm} / \mathrm{s}$, and LAD $\geq 42 \mathrm{~mm}$ ) for predicting CPAF are shown in Table 4 . The specificity of the LAD finding alone was not high (approx. 70\%), but that of combination findings of SEC and/or LAAF with LAD increased to $>90 \%$. The sensitivity of LAD was $60 \%$, while that of SEC, LAAF, or their combination was $<40 \%$.

\section{Discussion}

Previous studies have shown that some clinical parameters, such as age, frequent premature atrial contraction, mitral regurgitation, an increased left atrial diameter or volume, an increased left ventricular diameter, and a large cerebral lesion diameter, are associated with AF or PAF [12-17]. However, no single parameter may be useful for identifying patients at increased risk of CPAF in patients with ESUS because sensitivities and specificities of these parameters are not sufficiently high. With regard to echocardiographic findings, only a few studies have examined their association with CPAF as follows. Enlargement of the LAD [12], a low left atrial appendage ejection fraction [14, 15], and a low LAAF [14] have been shown to be predictors for PAF. In the present study, we also showed that enlargement of LAD by TTE and low LAAF by TEE were associated with PAF in patients with ESUS. We also showed that the presence of SEC was associated with CPAF. This is the first report to show that SEC can predict CPAF in patients with ESUS. However, SEC is reportedly associated with left atrial thrombus and embolism in patients with AF [18]. 
In our study, the specificity for detecting CPAF with an increased LAD by TTE was low (70\%). However, a finding of LAD with that of SEC and/or LAAF dramatically increased the specificity. The specificity of three combined parameters (SEC, LAAF $<46.9 \mathrm{~cm} / \mathrm{s}$, or LAD $\geq 42$ $\mathrm{mm}$ ) was $99 \%$ in our study. Skaarup et al. [15] also showed that a combination of parameters (left atrial appendage ejection fraction $<45 \%$, minimal left atrial volume $>23 \mathrm{~mL}$, and age $\geq 60$ years) could be used to detect CPAF with a sensitivity of $95.0 \%$. In patients with ESUS, detection of the embolic source is crucial for preventing recurrent stroke. Although a secondary prevention strategy of ESUS has not been established [19], we select anticoagulants when we strongly suspect the presence of PAF. The present findings of TEE in addition to TTE could contribute to selecting medication because the specificity became high after adding TEE findings.

The sensitivity of each single parameter of echocardiographic findings or that of their combination was not high in the present study. This finding is consistent with previous studies $[14,15]$. The reason for this low sensitivity might be partly because CPAF could be overlooked. Better devices and/or methods, including an insertable cardiac monitor, are required for evaluating the sensitivity for detecting CPAF.

Our study has several limitations. First, this was a retrospective study based on medical records from a single center. However, the rate of lost to follow-up was low (3\%). Second, our study has a possible sample selection bias. Although $76 \%$ of patients with ESUS underwent TEE, very old patients or those with a critical condition were excluded because of its invasiveness. Third, we did not assess the interrater reliability of echocardiography, although skilled ultrasonographers and neurologists, respectively, performed TTE and TEE. Fourth, CPAF might exist during the admission, although ECG monitoring or Holter ECG were performed in all patients. However, we could not completely exclude CPAF at the moment. Finally, an attempt to detect CPAF was not systematically performed. However, our hospital and affiliated clinics shared a consensus protocol for detecting CPAF. Therefore, CPAF might have been missed in some patients, and the detection rate of CPAF might have been underestimated.

\section{Conclusion}

The present study shows that findings of TEE in addition to those of TTE may be useful for identifying patients at increased risk of CPAF in cases of ESUS.

\section{Acknowledgments}

We thank Drs. Shota Sakai and Shunsuke Kimura for creating a database of patients with stroke in the Steel Memorial Yawata Hospital. We thank Ellen Knapp, PhD, from Edanz Group (www.edanzediting.com/ac) for editing a draft of this manuscript.

\section{Statement of Ethics}

This retrospective study was approved by our institutional ethics review board, and informed consent for each patient was waived because of the retrospective nature of this study.

\section{Disclosure Statement}

The authors have no conflicts of interest to declare. 
Ohya et al.: TEE Findings for Predicting CPAF in ESUS

\section{Funding Sources}

This research was not supported by any funding sources.

\section{Author Contributions}

Yuichiro Ohya and Masato Osaki designed the study and wrote the initial draft of the manuscript. Shuji Arakawa contributed to analysis and interpretation of data, and assisted in the preparation of the manuscript. All other authors contributed to data collection and interpretation, and critically reviewed the manuscript. All authors approved the final version of the paper and agree to be accountable for all aspects of the work in ensuring that questions related to the accuracy or integrity of any part of the work are appropriately investigated and resolved.

\section{References}

1 Hart RG, Diener HC, Coutts SB, Easton JD, Granger CB, O’Donnell MJ, et al.; Cryptogenic Stroke/ESUS International Working Group. Embolic strokes of undetermined source: the case for a new clinical construct. Lancet Neurol. 2014 Apr;13(4):429-38.

2 Sanna T, Diener HC, Passman RS, Di Lazzaro V, Bernstein RA, Morillo CA, et al.; CRYSTAL AF Investigators. Cryptogenic stroke and underlying atrial fibrillation. N Engl J Med. 2014 Jun;370(26):2478-86.

3 Seet RC, Friedman PA, Rabinstein AA. Prolonged rhythm monitoring for the detection of occult paroxysmal atrial fibrillation in ischemic stroke of unknown cause. Circulation. 2011 Jul;124(4):477-86.

4 Katsanos AH, Bhole R, Frogoudaki A, Giannopoulos S, Goyal N, Vrettou AR, et al. The value of transesophageal echocardiography for embolic strokes of undetermined source. Neurology. 2016 Sep;87(10):988-95.

5 Katsanos AH, Patsouras D, Tsivgoulis G, Kosmidou M, Katsanos KH, Kyritsis AP, et al. The value of transesophageal echocardiography in the investigation and management of cryptogenic cerebral ischemia: a singlecenter experience. Neurol Sci. 2016 Apr;37(4):629-32.

6 Saric M, Armour AC, Arnaout MS, Chaudhry FA, Grimm RA, Kronzon I, et al. Guidelines for the use of echocardiography in the evaluation of a cardiac source of embolism. J Am Soc Echocardiogr. 2016 Jan;29(1):1-42.

7 Santiago D, Warshofsky M, Li Mandri G, Di Tullio M, Coromilas J, Reiffel J, et al. Left atrial appendage function and thrombus formation in atrial fibrillation-flutter: a transesophageal echocardiographic study. J Am Coll Cardiol. 1994 Jul;24(1):159-64.

8 Lang RM, Bierig M, Devereux RB, Flachskampf FA, Foster E, Pellikka PA, et al.; Chamber Quantification Writing Group; American Society of Echocardiography's Guidelines and Standards Committee; European Association of Echocardiography. Recommendations for chamber quantification: a report from the American Society of Echocardiography's Guidelines and Standards Committee and the Chamber Quantification Writing Group, developed in conjunction with the European Association of Echocardiography, a branch of the European Society of Cardiology. J Am Soc Echocardiogr. 2005 Dec;18(12):1440-63.

9 Hanley PC, Tajik AJ, Hynes JK, Edwards WD, Reeder GS, Hagler DJ, et al. Diagnosis and classification of atrial septal aneurysm by two-dimensional echocardiography: report of 80 consecutive cases. J Am Coll Cardiol. 1985 Dec;6(6):1370-82.

10 Mas JL, Arquizan C, Lamy C, Zuber M, Cabanes L, Derumeaux G, et al.; Patent Foramen Ovale and Atrial Septal Aneurysm Study Group. Recurrent cerebrovascular events associated with patent foramen ovale, atrial septal aneurysm, or both. N Engl J Med. 2001 Dec;345(24):1740-6.

11 Pepi M, Evangelista A, Nihoyannopoulos P, Flachskampf FA, Athanassopoulos G, Colonna P, et al.; European Association of Echocardiography. Recommendations for echocardiography use in the diagnosis and management of cardiac sources of embolism: european Association of Echocardiography (EAE) (a registered branch of the ESC). Eur J Echocardiogr. 2010 Jul;11(6):461-76.

12 Muscari A, Bonfiglioli A, Faccioli L, Ghinelli M, Magalotti D, Manzetto F, et al. Usefulness of the MrWALLETS scoring system to predict first diagnosed atrial fibrillation in patients with ischemic stroke. Am J Cardiol. 2017 Apr;119(7):1023-9.

13 Gladstone DJ, Dorian P, Spring M, Panzov V, Mamdani M, Healey JS, et al.; EMBRACE Steering Committee and Investigators. Atrial premature beats predict atrial fibrillation in cryptogenic stroke: results from the EMBRACE trial. Stroke. 2015 Apr;46(4):936-41.

14 Shimizu T, Takada T, Shimode A, Fujita Y, Usuki N, Kato B, et al. Association between paroxysmal atrial fibrillation and the left atrial appendage ejection fraction during sinus rhythm in the acute stage of stroke: a transesophageal echocardiographic study. J Stroke Cerebrovasc Dis. 2013 Nov;22(8):1370-6. 
15 Skaarup KG, Christensen H, Høst N, Mahmoud MM, Ovesen C, Olsen FJ, et al. Diagnosing paroxysmal atrial fibrillation in patients with ischemic strokes and transient ischemic attacks using echocardiographic measurements of left atrium function. Am J Cardiol. 2016 Jan;117(1):91-9.

16 Thijs VN, Brachmann J, Morillo CA, Passman RS, Sanna T, Bernstein RA, et al. Predictors for atrial fibrillation detection after cryptogenic stroke: results from CRYSTAL AF. Neurology. 2016 Jan;86(3):261-9.

17 Sudacevschi V, Bertrand C, Chadenat ML, Tarnaud C, Pico F. Predictors of occult atrial fibrillation in one hundred seventy-one patients with cryptogenic transient ischemic attack and minor stroke. J Stroke Cerebrovasc Dis. 2016 Nov;25(11):2673-7.

18 Black IW, Hopkins AP, Lee LC, Walsh WF. Left atrial spontaneous echo contrast: a clinical and echocardiographic analysis. J Am Coll Cardiol. 1991 Aug;18(2):398-404.

19 Mohr JP, Thompson JL, Lazar RM, Levin B, Sacco RL, Furie KL, et al.; Warfarin-Aspirin Recurrent Stroke Study Group. A comparison of warfarin and aspirin for the prevention of recurrent ischemic stroke. N Engl J Med. 2001 Nov; 345(20):1444-51. 